\title{
LA REDEFINICIÓN DE LA POLÍTICA ESPAÑOLA DE SEGURIDAD Y DEFENSA PARA LA SEGUNDA DÉCADA DEL SIGLO XXI: BORRÓN Y CUENTA NUEVA
}

\author{
Javier Ignacio García ${ }^{1}$ \\ IE University/UNISCI
}

\begin{abstract}
Resumen:
El artículo examina la situación de la política de Seguridad y Defensa de España al comienzo de la segunda década del siglo XXI y la necesidad de su redefinición a partir de la experiencia de los últimos diez años. El trabajo analiza la política de los distintos gobiernos durante este periodo, los constantes cambios que ha sufrido, la ausencia de consenso entre las fuerzas políticas, el rechazo social a algunas decisiones y la falta de coherencia y visión de largo plazo de otra. Se estudia el giro estratégico de la política Exterior y de Seguridad durante la segunda legislatura del Presidente Aznar -dando prioridad absoluta a la relación con Estados Unidos tras el 11S-, y el rechazo popular y electoral a esta decisión. A continuación se repasa cómo ha evolucionado la política de Seguridad y Defensa de los gobiernos del Presidente Rodríguez Zapatero, desde la simbólica retirada de Iraq hasta la decisión de participar junto a Estados Unidos en el escudo antimisiles de la OTAN. Una política de Seguridad caracterizada por su subordinación a la política interna, por su obsesión por la imagen y por las repercusiones sobre la opinión pública, por la improvisación y por la falta de coherencia. Finalmente, el artículo se ocupa de la Estrategia Española de Seguridad, anunciada en 2008 y aprobada al final de la legislatura, sin consenso con otras fuerzas políticas y sin tiempo para implementarse. Se analiza su contenido y su posible utilidad como base de la política de Seguridad y Defensa de los próximos años.
\end{abstract}

Palabras clave: Política de Seguridad y Defensa, Política Exterior de España, Estrategia Española de Seguridad.

Title in English: "Redifining Spain's Security and Defense Policy for the second Decade of the XXIst Century: Wiping the Slate clean".

\begin{abstract}
:
This article deals with the Spanish Security and Defense Policy at the beginning of the second decade of the XXI century, and the need to redefine it from the experience during the last ten years. The paper analyzes the different policies governments have followed during this period with constant changes, lack of consensus among political forces, social rejection and lack of long-term strategy. It also studies the strategic shift in the Foreign and Security Policy during the second term of the Aznar period, when the relationship with the U.S. became the absolute priority after the 11-S, prompting both the popular rejection and the electoral defeat of the Popular Party. Furthermore, the article studies the evolution of the Security and Defense policy of the government of President Rodriguez Zapatero, starting from the symbolic withdrawal from Iraq up to the decision to take part with the United States in the NATO missile shield. This security policy was characterized by its subordination to domestic politics, obsession with public opinion and public image, improvisation and incoherence. Finally, the article deals with the Spanish Security Strategy approved at the end of the current legislature (although announced in 2008) without both political consensus and enough time to be implemented. We analyze its contents and potential usefulness as a basis for the Security and Defense policy in the coming years.
\end{abstract}

Keywords: Security and Defence Policy, Spanish Foreign Policy, Spanish Security Strategy.

Copyright $\odot$ UNISCI, 2011.

Las opiniones expresadas en estos artículos son propias de sus autores, y no reflejan necesariamente la opinión de UNISCI. The views expressed in these articles are those of the authors, and do not necessarily reflect the views of UNISCI.

\footnotetext{
${ }^{1}$ Javier Ignacio García González es profesor de Relaciones Internacionales, Globalización y Ciencias Políticas en la Escuela de Comunicación de la IE University. Es investigador senior UNISCI.

Dirección: Escuela de Comunicación, Universidad IE, Campus de Santa Cruz la Real, C/ Cardenal Zúniga 12, 40003 Segovia, España. Email: javier.garcia@ie.edu.

http://dx.doi.org/10.5209/rev_UNIS.2011.v27.38145
} 


\section{Seguridad y Defensa en España; un territorio propicio para el enfrentamiento partidista}

En noviembre del año 2001 el Ministro de Defensa Español informaba al Congreso de los Diputados de la oferta de tropas que el Gobierno había hecho a Estados Unidos para colaborar en las operaciones militares de ese país en Afganistán, como respuesta a la ataques del 11 de septiembre. Se trataba de unos 1.000 efectivos de fuerzas terrestres, navales y aéreas, puestos a disposición de Estados Unidos como "contribución española a la coalición internacional contra el terrorismo"2. Como ocurrió con las ofertas de otros países, los norteamericanos decidieron no emplear estás unidades en las operaciones de combate que llevaron a cabo inicialmente y los primeros soldados españoles no llegarían a Afganistán hasta un poco más tarde, en enero de 2002, ya integrados en la International Security Assistance Force (ISAF), 117 centrado en la reconstrucción del país.

Casi diez años después, el 5 de octubre de 2011 el Presidente del Gobierno de España anunciaba en Bruselas ${ }^{3}$ que la base naval de Rota se convertiría en la nueva base permanente de los 4 buques de la US Navy dotados con el sistema AEGIS que serán una parte importante del componente naval del sistema de defensa antimisiles que la OTAN ha decido poner en marcha en Europa en los próximos años. La iniciativa, en palabras del Presidente, respondía a que "España es un miembro solidario y comprometido con la defensa colectiva de Europa y va a participar y a apoyar en esta iniciativa, que tiene como objetivo mejorar la defensa y la seguridad de nuestros ciudadanos"4. La intervención finalizaba informando que el principal partido de la oposición era conocedor de la decisión y había habido un diálogo positivo y constructivo en ese sentido.

Para alguien que desconozca la vida política española en los últimos diez años, estos dos acontecimientos, contados así, podrían dar la impresión que se corresponden con una política de Seguridad y Defensa bien definida, coherente y caracterizada por la continuidad, vinculada a una Política Exterior que también tiene esas características. Parecen decisiones de un Estado que tiene claramente delimitados sus intereses nacionales, sus prioridades y sus alianzas; decisiones que, por su importancia, el gobierno comparte con las fuerzas políticas de la oposición, sin que estén sometidas en exceso a la lucha partidista y al vaivén de los normales cambios de gobierno en cualquier país democrático, es decir, una política de Seguridad y Defensa que se considere una "Política de Estado".

Como bien sabe cualquiera que haya conocido la vida política española, esta supuesta impresión nada tiene que ver con la realidad. Todo lo contrario. Durante la última década hemos asistido en España tal vez al periodo más convulso de nuestra política de Seguridad y Defensa desde la llegada de la democracia -con permiso de la etapa del referéndum de la OTAN-. Una etapa marcada por enormes discrepancias y enfrentamientos entre las fuerzas

\footnotetext{
${ }^{2}$ Congreso de los Diputados, Comisión de Defensa: Comparecencia del Ministro de Defensa (Trillo-Figueroa), Diario de Sesiones, Comisiones, VII Legislatura, $n^{\circ}$ 376, 27 de noviembre de 2001, p. 12348. Según la información del Ministro de Defensa, España ofreció a Estados Unidos tras los ataques del 11-S una unidad de operaciones especiales del Ejercito de Tierra y 8 helicópteros con un total de 235 personas, así como dos fragatas y un buque de apoyo logístico de la Armada, 8 aviones de transporte y uno de patrulla marítima del Ejército del Aire, que sumaban otros 736 efectivos.

${ }^{3}$ Gobierno de España: Palabras del presidente del Gobierno (Rodríguez Zapatero) después de su reunión con el secretario general de la OTAN y con el secretario de Defensa de Estados Unidos, Bruselas, 5 de octubre de 2011, en www.lamoncloa.gob.es/Presidente/Intervenciones/Otros/2011/prot2011105.htm

${ }^{4}$ Ibid.
} 
políticas, por falta de definición estratégica, con cambios de prioridades y de alianzas, así como por la utilización partidista y hasta personalista de la política Exterior y de Seguridad.

Las dos situaciones que se relatan al comienzo del texto, una vez se contextualizan, son precisamente ejemplos de esa falta de política de Seguridad y Defensa estable y con continuidad, de ausencia de una mínima visión compartida entre las fuerzas políticas que se alternan en el gobierno y de éstas con la misma sociedad a la que representan. En definitiva, son muestra de las diferencias a la hora de definir los intereses nacionales y los principios rectores de una política que, especialmente en los últimos tiempos, no ha sido prioritaria y se ha movido por la indeterminación.

Por una parte, la intervención del Ministro Federico Trillo-Figueroa en el Congreso se corresponde con los primeros movimientos del giro que el Gobierno conservador del Presidente Aznar dio a la política Exterior y de Seguridad española con motivo de los ataques del 11 de septiembre de 2001. A partir de ese momento, la relación transatlántica con Estados Unidos, aunque siempre ha estado entre las principales líneas de acción la Política Exterior española, se convirtió en la prioridad absoluta del Gobierno de España. El apoyo incondicional (al menos en lo político) a la postura de la administración Bush en la invasión de Iraq provocó un rechazo social y un enfrentamiento con otras fuerzas políticas que fue determinante para la inesperada victoria electoral del Partido Socialista en $2004^{5}$.

El segundo episodio citado, la comparecencia en la sede de la OTAN (octubre 2011) del Presidente Rodríguez Zapatero -líder del Partido Socialista en ese momento- para informar del despliegue en la base naval de Rota de los buques norteamericanos del escudo antimisiles de la OTAN, será buen ejemplo de la falta de coherencia y continuidad de la política de Seguridad y Defensa de un gobierno cuya primera decisión en este ámbito (2004) fue la retirada unilateral de las tropas españolas desplegadas en $\operatorname{Iraq}^{6}$, sin consultas con los aliados. Con esa decisión se daba cumplimiento a una promesa electoral, pero provocó una fuerte crisis diplomática y en las relaciones políticas con Estados Unidos, que tardarían años en normalizarse.

Entre la retirada de Iraq de 2004 y la participación junto a Estados Unidos en la defensa antimisil -en 2011- se han tomado toda una serie de decisiones que en el mejor de los casos marcaría una legítima evolución político-ideológica, pero que, a nuestro juicio, tiene más que ver con una falta de criterios claros y una política de elevada improvisación, en ocasiones dirigida por el cortoplacismo y la preocupación por los efectos sobre política interna y sobre la opinión pública y en otras por fogonazos repentinos de "responsabilidad internacional" y de solidaridad con los aliados. Sólo de esta forma podemos entender decisiones como la retirada unilateral de Kosovo y cómo se anunció, el incremento de la presencia militar en Afganistán, pero sin aceptar que se trata de la participación en una guerra, el impulso a los despliegues para la lucha contra la piratería en Somalia, la importante participación en algunas misiones de mantenimiento de la paz como la del Líbano, el despliegue de fuerzas para la formación de soldados somalíes en Uganda, o la participación en la guerra de Libia con restricciones -no autorizando misiones de ataque-, entre otras. Todo ello unido a una Política Exterior también errática y contradictoria en muchos aspectos, preocupada esencialmente por el "poder blando"

\footnotetext{
${ }^{5}$ Michavila, Narciso: "Guerra, terrorismo y elecciones: incidencia electoral de los atentados islamistas en Madrid”, Real Instituto Elcano, Documento de Trabajo 13/2005 (10-3-2005), p. 32. en www.realinstitutoelcano.org/documentos/180/Michavilapdf.pdf.

${ }^{6}$ La decisión, tomada una vez en el gobierno, estuvo precedida de una polémica por el "desplante" del entonces líder socialista ante el paso de la bandera norteamericana durante el desfile militar del Día de la Hispanidad, el 12 de octubre de 2003.
} 
-ejemplificado en la Alianza de Civilizaciones ${ }^{7}$ - y reticente a toda forma de "poder duro" que proporcionara credibilidad. Una acción exterior rodeada por la polémica y la falta de acuerdo con otras fuerzas políticas y mediantes la que España no ha acabado de encontrar su sitio en el mundo.

Como señalaba Günter Maihold en 2009 "España sigue buscando su lugar en la política internacional, no sólo para conseguir una adecuada percepción del país por parte de los demás actores, sino, tal vez, como consecuencia de una aportación insuficiente en relación con la sustantividad de las aportaciones que se esperan de ella para la solución de problemas" ". En definitiva, una política irrelevante en gran medida. No se ha mejorado en absoluto esta situación en los dos años posteriores. Como señalan otros autores, "lo que parece claro es que ha terminado un ciclo temporal en el que, desde hace al menos diez años, gobiernos muy distintos han intentado expandir la influencia de España en el mundo sobre unas bases -como le acaba de ocurrir a la economía- que se han demostrado poco sólidas"9.

Si nos circunscribimos exclusivamente al campo de la seguridad y la defensa, la aprobación en 2011 de la primera Estrategia Nacional de Seguridad, a menos de cinco meses del final de la legislatura y sin el consenso de otros grupos políticos, encaja también en esa concepción de escasa coherencia: se cierra un ciclo de gobierno con un documento que es propio de los comienzos de una legislatura, que incluye un importante contenido programático y una "visión" que un nuevo gobierno no tiene por qué aceptar. Sobre su contenido hablaremos más adelante.

\section{Seguridad y Defensa en la primera década del siglo XXI}

\subsection{La ausencia del consenso como rasgo característico}

La política de Seguridad y defensa de España en los primeros diez años del nuevo siglo se caracteriza claramente por la ausencia del consenso.

La política de seguridad y defensa en los países avanzados -como su política exteriorse debería situar dentro de las denominadas "políticas de Estado", es decir, pertenece al conjunto de cuestiones verdaderamente esenciales para las naciones desde una perspectiva histórica, cuyos efectos y resultados van más allá del corto plazo o de un gobierno en concreto, afectando a la posición e intereses permanentes del Estado y a su credibilidad internacional. Por su naturaleza, se considera que se trata de un ámbito de decisión que debe quedar fuera del debate político ordinario entre los partidos, y tiene que ser abordado con una visión más amplia, que aspire al mayor grado de consenso posible entre los distintos actores del sistema político. Se trata con ello de proporcionar una coherencia y estabilidad que beneficie la consecución de los grandes objetivos nacionales que se persiguen. El consenso interno acerca de estos objetivos, tanto entre las fuerzas políticas como en el conjunto de la sociedad, hace más fuerte a un país frente al resto del mundo. Como señalaba Andrés Ortega,

\footnotetext{
${ }^{7}$ Del Arenal, Celestino (2008): "Entre la afirmación de la dimensión normativa y el reforzamiento del pragmatismo: la política exterior y de seguridad de España en 2007”, en: Anuario CIDOB 2008, Barcelona, p.337.

${ }^{8}$ Maihold, Gunter (2010): “La política exterior y de seguridad española en 2009: la búsqueda del "sitio de España” en las relaciones internacionales”, en: Anuario CIDOB 2010, Barcelona, p.317.

${ }^{9}$ Molina, Ignacio y Tovar, Juan (2011): "El año que estalló la otra burbuja: la política exterior y de seguridad española en 2010”, en Anuario CIDOB 2011, Barcelona, p.272.
} 
si eso se lograra "España ganaría mucho, interna y externamente, como sabe muy bien cualquiera que haya pasado por una reunión o institución internacional" ${ }^{\text {"10 }}$.

Esta premisa no supone en absoluto que el diseño y la implementación de la política de seguridad y defensa esté fuera del escrutinio y crítica de la oposición o de la opinión pública. Sin embargo, se asume que la acción de los Estados en estos ámbitos suele presentar unas líneas de continuidad que responden a unos intereses permanentes, que no dependen del partido en el gobierno en cada momento, sino que tienen que ver con factores estructurales, con la historia, la geografía, la composición social o el desarrollo político, económico o cultural de ese Estado. Como señala Javier Solana, "La política exterior de un gran país, como el nuestro, no está sometida a cambios frecuentes. Nuestros intereses están definidos y España pertenece ya a las instituciones multilaterales fundamentales"11.

La existencia de esta continuidad no quiere decir tampoco que la política de los países sea algo invariable e inamovible, y, de hecho, hay cambios habitualmente. Resulta así perfectamente normal que gobiernos de distinta orientación ideológica pongan en práctica políticas exteriores con acentos diferentes o varíen las prioridades de actuación, pero normalmente serán aproximaciones distintas a esas cuestiones o intereses esenciales permanentes.

De mayor alcance serán los cambios a los que obliga la propia evolución del contexto internacional y no las diferencias de las fuerzas políticas. Las transformaciones de la sociedad internacional, a veces muy profundas y rápidas, obligan a la definición de nuevos objetivos, estrategias y políticas para dar respuesta a problemas o cuestiones que antes no existían o han cambiado radicalmente de importancia. Las alteraciones estructurales del sistema internacional, el impacto de fenómenos como la globalización -económica, social o políticao fenómenos como la actual crisis económica internacional, cuyas consecuencias no son meramente coyunturales, obligan a desarrollar nuevas líneas de acción o a la adaptación de las existentes. La consolidación de estos cambios como parte de esa "política de Estado" y darles continuidad, estabilidad y coherencia serán algunos de los desafíos a los que todos los países, también España, se enfrentan. En nuestro caso, podemos considerar la última década como perdida, pero la necesidad de ir por esta vía en el ámbito de la política de seguridad y defensa será cada vez más acuciante en el futuro -ya lo es hoy- a tenor de las transformaciones en curso del sistema internacional.

\subsection{Seguridad y defensa en el comienzo de la década: los efectos del 11-S en la política exterior española}

En general, la política exterior desarrollada por el Partido Popular entre su llegada al poder en 1996 y el año 2002 se puede definir por la continuidad respecto de las grandes líneas de acción de gobiernos anteriores, tanto en los objetivos y prioridades perseguidas, como en los distintos ámbitos de actuación ${ }^{12}$ : Europa -la Unión Europea- como gran prioridad, seguida de otras grandes áreas de interés de la acción exterior española como América Latina, el norte de África y el Mediterráneo y la relación transatlántica con Estados Unidos, por citar sólo las principales.

\footnotetext{
${ }^{10}$ Ortega, Andrés: "Moldear un nuevo consenso", FP Edición española (Febrero-Marzo 2005), p. 3.

${ }^{11}$ Solana, Javier: "España, 20 años de diplomacia”, Política Exterior, no 115, (Enero-Febrero 2007), p. 57.

${ }^{12}$ García Pérez, Rafael: "España en un mundo en cambio: a la búsqueda de la influencia internacional (19862002), en Pereira, Juan Carlos (co.) (2003): La política exterior de España (1800-2003), Barcelona, Ariel, p. 545.
} 
Sin embargo, será durante los dos últimos años en el poder del gobierno Aznar (20022004) cuando la política exterior y la política de seguridad y defensa se enfrenten a cambios más significativos, consecuencia de la evolución del sistema internacional ligada a los ataques del 11 de septiembre en Estados Unidos. En esa etapa, el gobierno de España dará un giro radical a su acción exterior, que afectará de forma esencial a los ejes principales que se habían mantenido hasta el momento. Dirigida personalmente desde la Presidencia del Gobierno, se acometió la tarea de cambiar las prioridades exteriores mantenidas hasta la fecha, mucho más allá de los cambios de aproximación que otros presidentes -incluso los propios gobiernos de Aznar hasta ese momento- habían realizado. Si bien es cierto que durante todo su mandato se había perseguido un alineamiento más estrecho con Estados Unidos ${ }^{13}$, se puede afirmar que desde finales del 2001 la voluntad de profundizar y reforzar la relación transatlántica con Estados Unidos pasará a ser el principal objetivo de la política exterior española, por encima de otros ejes de acción. Para algunos autores, este cambio de prioridades desde mediados de la segunda legislatura del presidente Aznar supuso la ruptura del consenso más importante de la historia de la política exterior y de seguridad de la democracia ${ }^{14}$.

Mucho tuvo que ver con ese giro la visión personal que el presidente tenía de la política exterior Española. Como señala Muñoz-Alonso ${ }^{15}$, a diferencia de otros presidentes que han tardado en situar a las cuestiones de política exterior en el mismo nivel de atención que otras cuestiones internas, Aznar desde el primer momento consideró este ámbito prioritario y de hecho, "fue siempre él quien se ocupó de diseñar las líneas maestras de la política exterior y quien fijó sus prioridades". En este sentido, el presidente resumía su objetivo en política exterior en una sola frase "Situar a España entre las mejores democracias del mundo", recuperando, según Muñoz-Alonso, una posición internacional perdida desde el Congreso de Viena.

Para el presidente Aznar, España ya estaba en lo que se puede considerar la normalidad internacional, -perteneciendo a las Organizaciones Internacionales que le interesaba, por ejemplo-, pero le faltaba la capacidad de "decidir" en el ámbito internacional, una capacidad de influencia que pese a su pertenencia a la OTAN y la UE no tenía. El primer paso para lograrla pasaba por asumir compromisos adecuados en la OTAN y por dejar de ser "el más grande de los pequeños" de la Unión Europea, para ser "el más pequeño de los grandes"16.

Defensor a ultranza del vínculo transatlántico entre Europa y Estados Unidos, Aznar consideró que los atentados del 11 de septiembre eran un momento de asumir responsabilidades frente a una amenaza como el terrorismo internacional, que atacaba los valores democráticos occidentales fundamentales. Era una ocasión para estar al lado de los norteamericanos, no sólo en los momentos inmediatamente posteriores a los ataques, sino también en sus implicaciones posteriores, como un aliado fiable. Respaldar a los Estados Unido en este momento, además de por pura convicción, suponía una oportunidad para ganar en visibilidad e influencia internacional en un mundo en el que la superpotencia de la Guerra Fría había salido de la indefinición estratégica de los años 90 y había decidido ejercer de potencia hegemónica tras ser atacada por un "nuevo" enemigo en su propio territorio. En definitiva, para ser grande había que estar al lado del grande, y una Unión Europea que en aspectos de política exterior y militares no tenía nada de "Unión", poco podía ofrecer a

\footnotetext{
${ }^{13}$ Ibid., p. 547.

${ }^{14}$ Del Arenal, Celestino: "La política exterior del gobierno socialista”, Política Exterior no 100 (Julio-Agosto 2004), p. 112.

${ }^{15}$ Muñoz Alonso, Alejandro (2007): España en primer plano. Ocho años de política exterior (1996-2004), Madrid, Gota a gota, pp. 92-97.

${ }^{16}$ Ibid.
} 
España en este sentido. En este contexto se debe entender, a nuestro juicio, la postura del Presidente Aznar ${ }^{17}$ de respaldo a norteamericanos y británicos respecto del problema de Iraq. Como señala Sahagún, el gobierno de Aznar "daba por bueno e inevitable el orden mundial basado en la hegemonía única de Estados Unidos y apoyó firmemente su nueva estrategia de seguridad"18.

Esta apuesta estratégica suponía un cambio de prioridades sin precedentes en la política exterior española. La relación transatlántica con Estados Unidos se configuraba como el eje prioritario, por delante de Europa, rompiendo así el tradicional consenso que había existido entre las distintas fuerzas políticas y la sociedad española en ese sentido.

Finalizadas las operaciones militares que acabaron con el régimen de Sadam Hussein, el gobierno español envió un contingente de alrededor de mil trescientos soldados en la fuerza multinacional de ocupación de Iraq, cuya misión era contribuir a la seguridad y hacer posible su reconstrucción. El despliegue de fuerzas en este caso se hizo con el respaldo legal de la resolución 1483 (2003), del Consejo de Seguridad de Naciones Unidas, de 22 de mayo, que "hace un llamamiento a los Estados miembros y las organizaciones internacionales para que... contribuyan a que existan en Iraq condiciones de estabilidad y seguridad de conformidad con la presente resolución", y con el de la resolución 1511 (2003), de 16 de octubre, que "autoriza a una fuerza multinacional bajo mando unificado a que tome todas las medidas necesarias para contribuir al mantenimiento de la seguridad y la estabilidad en Iraq" e "insta a los Estados miembros a que presten asistencia ..., incluso fuerzas militares, a la fuerza multinacional". Esta operación se realizó con un fuerte rechazo tanto de las fuerzas políticas de la oposición como de la opinión pública española.

Este último aspecto será fundamental. El giro en la política exterior fue rechazado desde el primer momento por la opinión pública, que no entendió o no compartió la apuesta estratégica del Presidente Aznar. Tal vez se le explicó mal o se hizo tarde, cuando ya estaba indisolublemente relacionado la guerra de Iraq, que se percibía como injusta e injustificada ya desde sus comienzos. Sea como fuere, la postura de respaldo a Estados Unidos en ese asunto se percibió como una adhesión incondicional al Presidente George W. Bush -cuando no una sumisión-, y se encontró con un rechazo ciudadano masivo en las encuestas (con porcentajes por encima del $80 \%$ en contra) y también en la calle, con importantes movilizaciones ciudadanas que no se recordaban desde la época del referéndum de la OTAN, con presencia de votantes de todo el espectro político.

Pese a este rechazo social generalizado a la postura sobre Iraq, el conjunto de las encuestas preelectorales de 2004 no mostraba que se fuera a producir un castigo determinante en las elecciones generales del 14 de marzo de ese año. Un tema básicamente de política exterior -aunque muy controvertido- no parecía suficientemente importante como para modificar la intención de voto de manera significativa. Sin embargo, los atentados del 11 de marzo en Madrid -casi 200 muertos y un millar de heridos-, tuvieron efectos inesperados sobre las votaciones de tres días después. La vinculación que la opinión pública percibió entre la "participación" de las tropas españolas en la guerra de Iraq y los atentados -atribuidos a un grupo islamista- tuvo finalmente mucho que ver con los movimientos de votos que

\footnotetext{
${ }^{17}$ Con posterioridad a la salida del gobierno del Partido Popular se han conocido las discrepancias dentro del propio gobierno y del Partido Popular acerca de la decisión del Presidente Aznar de respaldar incondicionalmente a Estados Unidos en el tema de Iraq, discrepancias que en ningún momento se dieron a conocer, manteniendo, al contrario, unidad de acción alrededor del liderazgo del jefe del ejecutivo y del partido.

${ }^{18}$ Sahagún, Felipe (2004): "Política exterior y de seguridad de España en 2004", en: Anuario Internacional CIDOB 2004, Barcelona, p. 265.
} 
provocaron la derrota electoral del Partido Popular y el inesperado triunfo del Partido Socialista en marzo de $2004^{19}$.

Los resultados electorales pondrían fin al giro de la acción exterior española que Aznar había diseñado, basado en un mundo surgido del 11-S en el que Estados Unidos asumía, porque creía que era capaz, un papel de hegemón global. España debía tener un papel destacado en ese nuevo orden. El tiempo ha demostrado que esa visión de una sociedad internacional caracterizada por la hegemonía de Estados Unidos ha quedado bien lejos de la realidad. A finales de la década nos estamos moviendo en un mundo mucho más complejo y multipolar, que la propia administración del Presidente Obama reconoce y con la que encaramos una segunda década del siglo que se podría describir como el Post-American World que describe en su bestseller Fareed Zakaria ${ }^{20}$ o como el Non-Polar World del que habla Richard N. Haass ${ }^{21}$. En todo caso, un mundo diferente del que parecía conformarse al principio de la década, y que será el escenario en el que el nuevo gobierno del Partido Socialista tuvo que poner en marcha su política de seguridad y defensa.

\subsection{La política de seguridad y defensa en los gobiernos socialistas de Rodríguez Zapatero}

Las imágenes ya citadas del presidente Rodríguez Zapatero anunciando la retirada unilateral inmediata de las tropas desplegadas en Iraq -nada más llegar al poder en 2004-, junto a la comparecencia del mismo presidente explicando el incremento de tropas norteamericanas en España como contribución nacional a la defensa antimisil de la OTAN -a punto de abandonar el gobierno en 2011-, son un buen resumen, a nuestro juicio, de cómo ha sido la política de seguridad y defensa desarrollada por los distintos gobiernos del Partido Socialista en las dos últimas legislaturas.

Una política que consideramos ha estado caracterizada por un alto grado de incoherencia, con decisiones contradictorias en no pocas ocasiones, con falta de estrategia y de objetivos claros y consensuados y de perfil bajo en todo lo que no fue el rechazo a la postura pro-norteamericana del Presidente Aznar.

Una política que ha sido activa y solidaria en algunos frentes (liderazgo en la lucha contra la piratería y en la formación de tropas somalíes, incremento de la presencia en Afganistán, compromiso con la misión de Mantenimiento de la Paz en el Líbano, participación en la guerra en Libia, etc.), pero que en muchos casos resulta compleja de entender como parte de un diseño coherente y consistente de política de seguridad y defensa.

Una política, finalmente, que ha estado condicionada en exceso por la preocupación por las repercusiones que las decisiones en temas de seguridad y defensa podían tener sobre la

\footnotetext{
${ }^{19}$ Michavila, Narciso, op. cit., p. 32-33. El citado estudio concluye que la incidencia de los atentados "aun siendo pequeña en términos porcentuales, fue determinante para cambiar el resultado final". "El resultado final puede cifrarse en la activación de un millón setecientos mil votantes que acudieron a votar movidos por los atentados y por el clima generado, unido a trescientos mil electores que dejaron de votar por la misma causa... La conversión de voto fue menor, algo más de un millón de electores, pero sus efectos electorales mayores al tratarse del trasvase de un partido a otro".

${ }^{20}$ Zakaria, Fareed (2009): El mundo después de USA, Madrid, Espasa. El libro comienza: "Este libro no trata del declive de América, sino del ascenso de todos los demás"

${ }^{21}$ Haass, Richard N.: "The Age of Nonpolarity; What Will Follow U.S. Dominance", Foreign Affairs, (MayJune 2008). Para el autor, la principal característica de las relaciones internacionales del siglo XXI es que "el mundo está dominado no por uno o dos o incluso varios Estados, sino por docenas de actores que poseen y ejercen distintos tipos de poder".
} 
política interna española en general y sobre la opinión pública en particular; un rasgo que estuvo presente en la retirada de tropas y en las declaraciones públicas posteriores sobre Iraq, en la política informativa sobre la situación en Afganistán, y que ha caracterizado de forma transversal la gran mayoría de iniciativas en el campo de la seguridad y la defensa, tal es el caso de la retirada de Kosovo o el lanzamiento de la misión contra la piratería.

La llegada al poder del partido socialista en 2004 supuso la puesta en marcha de una política exterior y de seguridad que se podía definir de una manera simple por un intento de "hacer lo contrario" de lo que hizo Aznar. Para los responsables de la política exterior del Partido Socialista, la política exterior durante la primera legislatura de Rodríguez Zapatero (2004-2008) había "conseguido reparar, desideologizar y reformar nuestra acción internacional para recuperar los valores y ejes tradicionales, junto a algunos nuevos, adaptándolos a los intereses de España ante los retos y oportunidades del mundo hoy, frente a la estrategia negativa y unilateral del Partido Popular, que ahogó las líneas maestras de una política exterior labrada durante más de dos décadas" 22 .

Sin ninguna sorpresa, la percepción del principal partido de la oposición y su balance de la política exterior y de seguridad eran bien distintos y no había supuesto, en absoluto, ningún tipo de vuelta al consenso o al acuerdo básico entre las principales fuerzas políticas. Así, su programa electoral para las elecciones de 2008 planteaba en su punto 540 la reconstrucción del consenso "para hacer de la política exterior un objetivo compartido al servicio de los valores de nuestra democracia y de sus intereses".

Los primeros años de la política de seguridad y defensa de los gobiernos socialistas estuvieron marcados por las decisiones alrededor del conflicto de Irak. El domingo 18 de abril de 2004, el día después de tomar posesión como Presidente del Gobierno, Rodríguez Zapatero compareció públicamente para anunciar que había dado la orden de al Ministro de Defensa de retirar las tropas españolas desplegadas en Irak "en el menor tiempo posible y con la mayor seguridad posible".

Se adelantaba así el plazo que se había fijado para decidir sobre la presencia de las tropas, inicialmente hasta el 30 de junio. Según la declaración oficial, se debía a que no era previsible en ese plazo una resolución de la ONU que se ajustara a las condiciones expuestas antes de las elecciones por el PSOE. Considerando que esas condiciones suponían que la ONU tenía que hacerse cargo de toda la misión de reconstrucción de Irak y asumir el mando militar de la operación y de las decenas de miles de soldados norteamericanos en ese territorio -algo imposible de imaginar- se trataba de una decisión tomada. Otra cuestión eran los plazos y las formas, sobre los que no se fue claro en los días posteriores, ni tan siquiera en la sesión de investidura del Presidente en el Parlamento ${ }^{23}$. El hostigamiento que sufrían las tropas durante las últimas semanas en Irak, con frecuentes ataques a instalaciones y fuerzas, tal vez tuvo algo que ver, como recogieron algunos medios de comunicación.

La decisión de retirada en los términos en los que se planteó, abrió inmediatamente una enorme brecha en la relación con Estados Unidos, que se mantuvo abierta prácticamente hasta la llegada de la nueva administración Obama en 2009. La retirada era un compromiso

\footnotetext{
${ }^{22}$ Valenciano, Elena (Secretaria de Relaciones Internacionales del PSOE): “Rumbo al futuro: una España más fuerte en un mundo más justo”, Intervención en el Real Instituto Elcano, 14 de febrero de 2008. http://www.realinstitutoelcano.org/wps/portal/rielcano/contenido?WCM_GLOBAL_CONTEXT=/elcano/elcano es/zonas_es/00023.

${ }^{\overline{23}}$ Ruiz Miguel, Carlos: “La retirada española de Irak: significado y consecuencias”, Real Instituto Elcano, ARI $n^{o} 81 / 2004$ (2004), p. 2.
} 
recogido en el programa electoral, efectivamente, pero la precipitación al anunciarla, sin consultar ni coordinarla con Estados Unidos ni con otros países afectados con los que se compartían responsabilidades, como Polonia o varios países latinoamericanos, fue recibida como una traición a los aliados y como una cesión al chantaje del terrorismo. Ni la forma de retirar las tropas de Irak, ni las frecuentes declaraciones de miembros del gobierno recriminando la política del Presidente Bush, con una constante referencia a la "guerra ilegal" que había empezado Estados Unidos, ayudaron a que el gobierno norteamericano mostrara buena disposición para normalizar las relaciones.

Por parte española, en lo que quería ser una señal importante, casi una compensación por la retirada de Iraq, se reiteró el compromiso con las operaciones militares aliadas en Afganistán. Con el tiempo no sólo se ha mantenido la presencia de tropas en aquel país, sino que el número de efectivos se ha incrementado en sucesivas ocasiones hasta alcanzar prácticamente los 1.500 hombres y mujeres, siempre bajo mando de la OTAN, y con un perfil bajo cara a la opinión pública. La falta de información y transparencia sobre el uso de la fuerza en combate de las tropas ha sido una de las constantes durante los últimos años, intentando reducir la percepción pública de que se está interviniendo en un conflicto armado.

En todo caso, la retirada de las tropas de Irak se convirtió en el gran icono de la política exterior y de seguridad de Zapatero en el que se ponían de manifiesto buena parte de lo que eran las líneas maestras del nuevo gobierno en este ámbito: ruptura radical y distanciamiento de la política exterior y de seguridad del Presidente Aznar; vuelta a la prioridad Europea con acercamiento al eje franco-alemán también en temas de seguridad -reforzamiento de la Política Exterior y de Seguridad Común-; debilitamiento de la relación transatlántica con Estados Unidos; apuesta por el multilateralismo y por el papel de Naciones Unidas. En suma, para el que fuera el Ministro de Asuntos Exteriores durante la mayor parte de los gobiernos de Rodríguez Zapatero, era una "recuperación de una política de Estado", una "vuelta al consenso" y a los ejes clásicos de la política exterior de España ${ }^{24}$. Pasados los años, no podemos afirmar que ese propósito programático se haya cumplido, y además buena parte de esas apuestas iniciales se han desvelado como equivocadas.

El concepto de multilateralismo, tal vez el más utilizado por los responsables de la acción exterior española en los últimos años, también ha tenido su reflejo en el ámbito de la seguridad y la defensa. Los sucesivos gobiernos del Presidente Zapatero plantearon una política de "multilateralismo eficaz", que suponía la actuación en el ámbito exterior con pleno respeto a la legalidad internacional, el fomento del diálogo y el consenso internacional, el fortalecimiento de Naciones Unidas y la acción en el seno de otras Organizaciones Internacionales. En ese sentido, esta visión se concebía como una filosofía transversal que atraviesa toda la acción exterior, también la del ámbito de la seguridad y la defensa, pero sin poner excesivo énfasis en este aspecto ya que estaba más en concordancia con el objetivo de situar a España como referente de "poder blando", muy en la línea también de cierta corriente pacifista propia de la sociedad española.

Manteniendo la coherencia en este caso, el discurso multilateralista sí ha encontrado respuesta en la participación española en las Operaciones de Mantenimiento de la Paz lanzadas por Naciones Unidas, particularmente asumiendo un papel muy relevante con el despliegue de mas de 1.000 soldados en la misión UNIFIL en el Líbano -desempeñando

\footnotetext{
${ }^{24}$ Moratinos, Miguel Ángel: "Una nueva política exterior para España”, Política Exterior no 99, (Mayo-Junio 2004), p. 65.
} 
también el mando de la fuerza desde 2010-, o acogiendo en Valencia una nueva Base de Apoyo Logístico a las Misiones de Paz de las Naciones Unidas.

La "vuelta al corazón y a la razón del proyecto de construcción europea" 25 defendida por el gobierno de Zapatero también ha tenido su reflejo en el apoyo español al desarrollo de la Política Europea de Seguridad y Defensa (PESD). Un apoyo contribuyendo al establecimiento de sus capacidades militares (algunas nunca utilizadas como los Batlegroups), manteniendo su presencia en las operaciones en marcha y participando en otras nuevas como la misión de apoyo a las Naciones Unidas en el Congo en el año 2006 o la misión en el Chad en 2008-2009.

Mención especial merecen las operaciones de la Unión Europea de lucha contra la piratería en las costas de Somalia -operación Atalanta- y de entrenamiento de tropas somalíes en Uganda -EUTM Somalia-, en marcha respectivamente desde 2008 y desde 2010, en las que España ha tenido un papel determinante, coliderando la iniciativa junto a Francia en el primer caso, y liderando como primera nación marco en el segundo. Más allá de considerar si la presencia es Somalia debe estar entre las prioridades estratégicas de España o no, estás misiones sí han conseguido dar visibilidad exterior tanto a la Política Europea de Seguridad y Defensa como a la presencia de las Fuerzas Armadas españolas, en el marco de una misión militar poco convencional que se ocupa de los "nuevos riesgos", tan diferentes de los escenarios más clásicos de conflicto armado.

No obstante, el fortalecimiento de la PESD (actual Política Común de Seguridad y Defensa, PCSD) se encontró en estos años con serias dificultades derivadas de la falta de definición del proyecto europeo que siguió al fracaso de la Constitución, así como a los problemas de implementación que se están dando en la puesta en marcha de los nuevos mecanismos de acción exterior del Tratado de Lisboa. Todo ello en un contexto de crisis económica que ocupa prácticamente en exclusiva las agendas de unos dirigentes políticos europeos a los que con frecuencia se acusa precisamente de falta de liderazgo. Esta situación se ha traducido en el caso de España en una política también de perfil bajo, a veces sin rumbo fijo, vacilante en cuanto a la búsqueda de apoyos, sin gran protagonismo e iniciativa, predispuesta a la colaboración, pero sin pretender maximizar los intereses nacionales.

Por último, la participación española en las operaciones militares en Libia se puede encuadrar también en esa aproximación del "multilateralismo eficaz" en seguridad y defensa. La intervención, aunque finalmente ha estado a cargo de la OTAN, se encuentra amparada en origen por la Resolución 1973 (2011) de Naciones Unidas y en ella se invoca el principio de la Responsabilidad de Proteger ${ }^{26}$, en lo que constituye una importante muestra de eficacia de la organización, en línea con el fortalecimiento de esta institución que siempre mantuvo el Gobierno.

\footnotetext{
${ }^{25}$ Moratinos, Miguel Angel: Comparecencia del señor Ministro de Asuntos Exteriores y de Cooperación para informar sobre 'balance y perspectivas de la nueva política exterior de España', a petición propia, 2 de febrero de 2005, en: Real Instituto Elcano (2007): La política exterior de España. Balance y debates parlamentarios (20042008), p. 80, en http://www.realinstitutoelcano.org/wps/wcm/connect/80b584004f0195b987d6e73170baead1/PoliticaExteriorEsp ana 2004-2008.pdf?MOD=AJPERES.

${ }^{26}$ García González, Javier Ignacio: "La responsabilidad de proteger (R2P) y su puesta en práctica: instrumentos de prevención y uso de la fuerza”, en Requena, Miguel (ed.) (2011): Actas de las III Jornadas de Estudios de Seguridad, IUGM-UNED, Madrid, pp. 539-551.
} 
Aunque coherente en este sentido, en ella también se muestran buena parte de las contradicciones que han caracterizado la política de seguridad española de los últimos años. Hay una rápida decisión del gobierno para colaborar en la implementación de la resolución junto a los países de la coalición internacional, pero la participación miliar española se plantea estrictamente sobre la base de una interpretación literal y restrictiva de la resolución, mientras otros aliados asumen misiones propias de una interpretación mucho más laxa, siendo decisivos en la evolución del conflicto. Si gran parte de nuestros aliados ha llevado a cabo misiones ofensivas y de ataque a objetivos del régimen de Gadafi, asumiendo el mayor riesgo de perdidas propias o de daños no deseados sobre la población civil libia, la participación española se ha limitado a medios para las operaciones de embargo -con un riesgo de uso de la violencia muy limitado- y a aviones de combate para mantener una zona de exclusión aérea que el régimen de Gadafi no podía violar en la práctica ya desde los primeros días del conflicto. En definitiva, una intervención con importantes restricciones que es también ilustrativa del papel que se ha querido dar a España en el actual escenario internacional.

\section{La Estrategia Española de Seguridad de 2011: ipunto de partida o despedida y cierre de una visión de la política de seguridad y defensa?}

Desde mediados de 2011 España dispone de una Estrategia Nacional de Seguridad. El Consejo de Ministros del 24 de junio aprobó el documento titulado Estrategia Española de Seguridad: una responsabilidad de todos, y dispuso su remisión a las Cortes Generales. La Estrategia Española de Seguridad (EES) es el primer documento de este tipo realizado en este país y pretende poner en marcha un nuevo modelo de política de seguridad, similar al de otros Estados como el Reino Unido, Holanda, Francia, Estados Unidos o Canadá, donde estos informes cuentan con una mayor tradición.

El documento español tiene su origen en el discurso de investidura del Presidente Rodríguez Zapatero en el año 2008, en el que se compromete a remitir a las Cortes un documento con los objetivos, prioridades, dotaciones y medidas organizativas y presupuestarias que debería guiar la política de seguridad y defensa a medio plazo, en definitiva, lo que conocemos como una estrategia de seguridad y defensa.

El proceso de elaboración de la EES desde el compromiso en 2008 fue complicado. Las rivalidades entre ministerios dificultaron la redacción de la EES inicialmente y sólo cuando se toma la decisión de nombrar a Javier Solana responsable de la redacción del documento el proyecto avanza seriamente. No obstante, una vez se entrega el borrador al Presidente en noviembre de 2010, el documento quedó paralizado hasta junio de 2011. Como reflejaban las informaciones de prensa, "reposa en un cajón de un Zapatero muy alejado de su discurso de investidura y en una Moncloa centrada fundamentalmente en la crisis"

Su aprobación tan tardía viene acompañada de serias dudas sobre sus posibilidades de puesta en marcha dado lo avanzado de la legislatura y con una coyuntura nacional e internacional que difícilmente iba a poner a la EES en un lugar prioritario. La Estrategia Española de Seguridad llegaba por tanto lastrada con un déficit importante, y es que este tipo de documentos son propios de los comienzos de legislatura y tienen una carga de contenido

\footnotetext{
${ }^{27}$ Rubio, Mariela: "Solana recomienda a Zapatero crear un Consejo de Seguridad Nacional”, Cadena SER, 13 de abril 2011, en http://www.cadenaser.com/espana/articulo/solana-recomienda-zapatero-crear-consejo-seguridadnacional/csrcsrpor/20110413csrcsrnac_3/Tes/?print=1.
} 
programático que, como señala Fojón "no es transferible a otros ejecutivos, sin perjuicio de que permanezcan los elementos básicos que informan la estrategia"28. Si a esto añadimos que se trata de un documento no consensuado con el Partido Popular, principal partido de la oposición y que puede ocupar el gobierno apenas unos meses después de aprobarse la EES, nos encontramos con un documento cuya vida puede ser muy corta, al menos en su actual versión. Difícilmente cumplirá el propósito inicial de estar vigente unos diez años y revisarse más o menos a los cinco.

En todo caso, la aprobación de la EES supone un paso adelante con respecto a la situación desde la que se venía. Desde los 90, generalmente coincidiendo con los comienzos de la legislatura, el Presidente del Gobierno promulgaba la Directiva de Defensa Nacional como el documento en el que se recogían las líneas generales de la política de defensa y las directrices que se establecían para su desarrollo.

La Estrategia pretende dar un salto cualitativo sobre esa aproximación tradicional a la seguridad fundamentalmente desde la perspectiva de la defensa, los aspectos militares o los puramente de orden público. Se plantea así una visión moderna de la seguridad, una visión "integral" o "multidimensional" que no es exclusivamente militar o policial. Se considera que la seguridad en la actualidad no sólo es defensa u orden público -como se entendió durante mucho tiempo-, sino que tiene que ver con las funciones de muchos y variados organismos del Estado, tanto de la administración Central (Ministerios) como de las administraciones regionales (Comunidades Autónomas) y municipales. Incluso la EES también pretende integrar en la seguridad a la sociedad civil, a través del sector empresarial privado y también de organizaciones sociales de diverso tipo.

La EES sería el documento político principal, emitido desde Presidencia de Gobierno, de carácter integrador de todas las políticas que tienen relación con algún aspecto de la seguridad. La EES tendría que servir de marco para los documentos de segundo o tercer nivel que elaborarían los distintos Ministerios, y que desarrollarían los distintos aspectos que afectan a cada organismo directamente. Esto supondría la concentración de las grandes decisiones e iniciativas sobre seguridad en el ámbito de la Presidencia de Gobierno, yendo más allá del modelo de coordinación o cooperación entre ministerios que está vigente en la actualidad, aunque luego se descentralizara la ejecución.

En cuanto al contenido y estructura de la EES se trata de un documento moderno, pero no demasiado diferente de los documentos similares de los otros países citados y que recoge también la influencia de la Estrategia Europea de Seguridad de 2003 (actualizada en 2008) y del Concepto Estratégico de la OTAN aprobado en octubre de 2010, después de elaborarse en poco más de año y medio.

Partiendo de una visión de España como "Potencia Media", el catálogo de amenazas y riesgos es similar al que se recoge en los documentos similares de otros países (referidos a conflictos armados, terrorismo, crimen organizado, inseguridad económica y financiera, seguridad energética, proliferación, ciberamenazas, migraciones incontroladas, emergencias y catástrofes e infraestructuras), señalando tanto las amenazas "tradicionales" ligadas a la defensa y la seguridad interior, como otros riesgos de tipo económico -la actual crisis económica también tiene su reflejo en la EES-, infraestructuras y similares.

\footnotetext{
${ }^{28}$ Fojón, Enrique: "El inicio de un largo camino", Atenea. Seguridad y Defensa, año IV, n’ 29 (Septiembre 2011), p. 29.
} 
Es importante destacar que la EES se refiere tanto a amenazas al Estado como a las amenazas a los ciudadanos, en la línea de un planteamiento de Seguridad Humana, que está muy presente también en la Estrategia Europea de 2003 coordinada igualmente por Solana. Las amenazas que se recogen en el texto y los denominados "potenciadores del riesgo" (características de la sociedad internacional actual que no se interpretan directamente como amenazas, sino como situaciones o fenómenos que pueden incrementar o reducir la importancia de esa amenazas o la forma de enfrentarnos a ellas), están en buena medida influidas por esa visión de la Seguridad Humana, centrada en la seguridad del individuo, no sólo la del Estado.

En cuanto a las respuestas a las amenazas (líneas de acción) ciertamente son bastante generales y poco novedosas o imaginativas. En muchas ocasiones parecen grandes afirmaciones de buenos deseos con un elevado grado de utopía, más que líneas de acción estratégica. A nuestro juicio, este aspecto sería uno de los más criticables de la EES, que puede dar la impresión más de un enunciado de amenazas que una reflexión sería de cómo enfrentarlas en el futuro.

El contexto internacional que recoge la Estrategia es también uno de los aspectos que en nuestra opinión puede dejar obsoleto el documento a corto plazo. Cuando el documento sitúa a España en el marco en el que debe desarrollar su política (Capítulo 2. la seguridad de España en el mundo), toma como punto de partida una concepción básicamente multipolar de la sociedad internacional, lo que consideramos acertado, pero explica las relaciones con aliados y con las organizaciones de las que formamos parte -UE, OTAN, Naciones Unidas, OSCE- en unos términos que ya podrían estar cambiando.

En este sentido, la EES no recoge las consecuencias de acontecimientos muy recientes que interpretamos como cambios de gran envergadura para el contexto de seguridad europeo, y que tienen que afectar directamente al diseño de cualquier estrategia de seguridad. Nos referimos concretamente a cómo la intervención militar en Libia ha puesto de manifiesto posiciones y situaciones que -sabiendo que no son nuevas- han alcanzado tal grado de madurez que pueden llegar a cuestionar las mismas bases de la seguridad euroatlántica.

Si alguna vez la tuvo, la credibilidad de la Política Común de Seguridad y Defensa (la anterior PESD), ha sido una de las grandes damnificadas del conflicto libio. La propia creación de una coalición internacional liderada por Francia y el Reino Unido dice muy poco de la confianza en una PCSD, que cumplió hace poco diez años y que, a pesar de las dificultades y las limitaciones, había sido capaz de poner en marcha hasta 22 misiones en zonas de conflicto. No lo ha sido en el caso de Libia, donde se veía afectada directamente la seguridad de la Unión en su conjunto, poniéndose de manifiesto una falta de unidad de esfuerzo y de credibilidad que tardará en superarse, si es que lo hace. La tardía y difícil entrada de la OTAN para hacerse cargo de las operaciones militares tampoco fue un ejemplo de cómo debe funcionar una alianza, desatando tensiones de fondo que no se acaban de resolver.

La escena de una votación en el Consejo de Seguridad de Naciones Unidas en la que el representante Alemán votaba en diferente sentido al de Francia y el Reino Unido no ha sido tampoco edificante ni esperanzadora. Más como excepción que como regla, el multilateralismo eficaz no se veía afectado por la abstención de un país central en Europa, junto a la de China y Rusia, pero una decisión de este tipo tiene una trascendencia mucho más allá del gesto. 
Tan sólo el compromiso mostrado por los norteamericanos desde el comienzo de la crisis, pese a ser un teatro donde los intereses directos son escasos, añade un dato de optimismo a la situación. Parece que la actual administración norteamericana ha resistido por el momento la tentación de desentenderse de una Europa que le aporta poco, que ya dejó hace tiempo de ser el escenario principal para su política exterior.

En definitiva, la Estrategia Española de Seguridad es un necesario e importante paso a la hora de definir una política de seguridad moderna y coherente para una potencia media como España, pero llega con notables limitaciones que pueden restarle valor, aunque no eliminan su utilidad. Independientemente de su vigencia a corto-medio plazo y de la aceptación o rechazo que pudiera tener hacia ella un nuevo gobierno, la elaboración de una estrategia de este tipo supone un esfuerzo intelectual relevante que no debe ser despreciado y debe servir como base para posteriores documentos del mismo nivel. 